\title{
Research on Data Transmission Optimization of Communication Network Based on Reliability Analysis
}

Hui Wang

Information College, Baoshan University, Baoshan, Yunnan 678000, China

E-mail: ynwhh@cumt.edu.cn

Keywords: vehicular ad-hoc network, ant colony algorithm, connectivity probability, routing algorithm

Received: August 17, 2020

\begin{abstract}
Firstly, this paper briefly introduces the vehicular communication network, vehicular ad-hoc network (VANET), and the ant colony algorithm for path search in the network. Then the ant colony algorithm is improved. A simulation experiment is carried out on the improved ant colony algorithm on the simulation platform, and it is compared with the traditional ant colony algorithm and the geographical locationbased routing algorithm. The results show that the network packet loss rate and time delay show a tendency of increasing first and then decreasing under the three algorithms with the increase of vehicle nodes in VANET; with the increase of nodes, the network reliability under the improved ant colony algorithm shows a tendency of increasing first and then being stable, and the network reliability under the other two algorithms shows a tendency of increasing first and then decreasing; under the same number of nodes, the packet loss rate and average delay are lower, and the reliability is higher under the improved ant colony algorithm.
\end{abstract}

Povzetek: Opisana je raziskava optimizacije prenosa podatkov po komunikacijskem omrežju na osnovi analize zanesljivosti prenosa.

\section{Introduction}

With the convenience of the wireless network, the Internet of things can connect different things anytime and anywhere to realize information interaction. The existence of the Internet of things has further facilitated our life [1]. In intelligent transportation, the interconnection of human-vehicle-road-environment is realized by the Internet of things to achieve the optimization of traffic resource allocation [2]. For the Internet of things, the data transmission quality of wireless communication network for information interaction can directly affect the service quality of the Internet of things. If the quality of data transmission in the communication network is poor, packet loss will often occur, or the data throughput will be small, so that the Internet of things cannot effectively carry out information exchange. More over due to the rule that data will be transmitted again if the transmission fails because of packet loss, the number of data transmission will increase, which will increase the energy consumption of the communication network [3]. Therefore, improving the quality of data transmission in the communication network is very important for the Internet of things. Kumar et al. [4] proposed a beacon independent geographic routing algorithm called BIIR which reduced the number of broadcasts to forward the data packets by making intelligent use of information collected by the vehicle during previous route discovery attempts for a destination and found through the simulation that the algorithm outperformed the existing beacon less routing protocols in terms of the average number of broadcasts per data packet forwarding, packet delivery ratio and end to end delay experienced by the data messages. Rahul et al. [5] proposed a routing algorithm based on genetic algorithm. They found that the algorithm had a great improvement in the execution time compared with the traditional serial algorithm and could be expanded with vehicles on the road to reduce the roadside units and provide a more extensive coverage. Ning et al. [6] proposed a reliable opportunistic routing algorithm based on probability prediction. The utility of relay vehicles in the candidate set was confirmed by predicting the change of the signal to interference plus noise ratio of the receiving end and packet queue length, so as to determine the route. Through simulation, they found that the algorithm had better routing performance than SRPE, ExOR, and GPSR routing algorithms. This paper briefly introduced the vehicular communication network, vehicular ad-hoc network (VANET) and the ant colony algorithm for path search in the network, improved the ant colony algorithm, carried out a simulation experiment on the improved ant colony algorithm on the simulation platform, and compared it with the traditional ant colony algorithm and the geographical location-based routing algorithm.

\section{Vehicle communication network}

With the help of wireless networks, different things form the Internet of things through information interaction, which greatly facilitates people's daily life. The communication network used for data transmission in the Internet of things of the intelligent transportation system is a vehicle communication network, also known as VANET [7]. The basic principle of VANET is: within the scope of communication, vehicles are connected to realize 
the interaction of the speed, location and detected environmental information to form a mobile network. The data transmission node in VANET is undertaken by vehicles, which makes the nodes in the network different from the network nodes in the general Internet of things. The nodes are not fixed but will constantly be moving and changing, which directly leads to the continuous change of the network topology structure of VANET, and the links between nodes may be disconnected at any time [8]. Moreover, as the node is the driving vehicle and the vehicle will be affected by the road environment in the process of driving, especially in the highway or urban road, the driving track of vehicles is limited, i.e., the moving track of network nodes are predictable.

The purpose of VANET is to realize the data interaction among vehicles, roads, and environment and make corresponding traffic resource allocation according to the collected data. However, the traffic environment in the road changes quickly because of the fast driving of vehicles. Therefore, VANET has strict requirements on the delay of data transmission. If data are lost because of the large time delay, the system cannot make a judgment in time. VANET needs to ensure the connectivity between nodes in data interaction. Nodes in the conventional Internet of things have fixed positions, and connectivity has been guaranteed in the design. However, most nodes in VANET are vehicles. Different vehicles have different speeds in the process of driving, and the connectivity between nodes will also change. Therefore, it is necessary to model and calculate the connectivity.

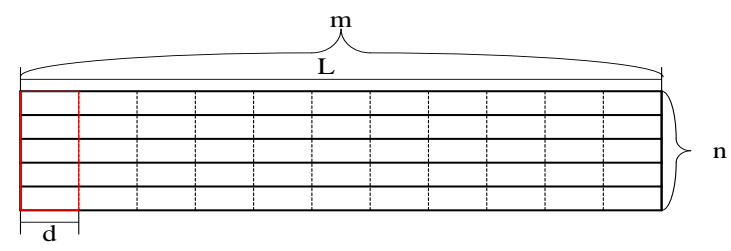

Figure 1: The schematic diagram of road division.

In this study, the connectivity model is built by dividing the road. As shown in Figure 1, the road is divided into $m$ grids (the red box in Figure 1 is a gird), there are ${ }^{n}$ lanes on the road, i.e., every grid has $n$ lanes, and the width of every cell is $n$. It is assumed that there is only one vehicle on every lane in every grid, and the communication radius of the vehicle is set as $R=m_{0} d$, where $m_{0}$ stands for the number of continuous grids.

The calculation formula of connectivity probability [9] is as follows:

$$
\left\{\begin{array}{l}
p_{1}=1-p_{2} \\
p_{2}=\sum_{k=\max \left(m-o, m_{0}\right)}^{\left.\max (m-\sigma o / n], m_{0}\right)}\{f(o, m)=k\} p\left\{g(m, k)>m_{0}\right\} \\
p\{f(o, m)=k\}=C_{m}^{k} \frac{C_{(m-k) n}^{o}}{C_{m n}^{o}} \sum_{k=\max \left(m-o, m_{0}\right)}^{m} C_{m}^{k}(-1)^{k} \frac{C_{(m-k) n}^{o}}{C_{m n}^{o}} \\
p\left\{g(m, k)>m_{0}\right\}=1-\frac{\sum_{i=k-m_{0}}^{\min \left\{k,(m-k) m_{0}\right\}} c[i]^{m-k}}{C_{m}^{k}}
\end{array}\right.
$$

where $p_{1}$ and $p_{2}$ are the connectivity probability and disconnectivity probability respectively, $p\{f(o, m)=k\}$ stands for the probability that there are no vehicles in $k$ road grids, $p\left\{g(m, k)>m_{0}\right\}$ stands for the probability that the number of continuous grids between $k$ road grids is larger than $m_{0}$, and symbols in the form of $C_{a}^{b}$ stand for the most probable number of selecting $b$ objectives from $a$ optional objectives for combination.

\section{Data transmission optimization algorithm}

VANET in the intelligent traffic system takes the vehicle as a node and transmits the information within the communication radius of the vehicle. The information interaction between vehicles constitutes the network. Naturally, for better relay transmission of information, the corresponding routing algorithm, or in other words, the data transmission path selection algorithm, is needed [10].

\subsection{Ant colony algorithm}

As an intelligent algorithm, the ant colony algorithm [11] is an imitation of the foraging phenomenon of an ant colony in nature, which is mostly used for issues such as TSP, scheduling optimization, and path selection. In this study, the data transmission optimization algorithm in VANET can be said to be a path selection problem, and moreover, the self-organization and dynamic variability of ant colony foraging is quite consistent with the characteristics of VANET. The ant colony algorithm is applied in VANET, and the process is as follows. Firstly, ants start from the source node and then select the next hop node from the neighbor candidate nodes according to the candidate probability. The calculation formula of candidate probability [12] is:

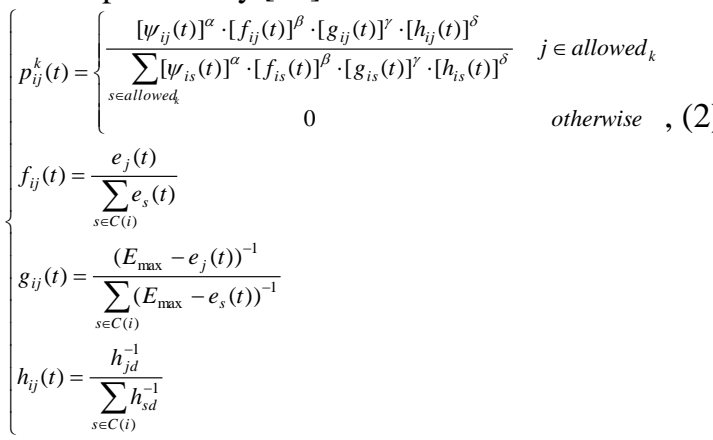

where $p_{i j}^{k}(t)$ represents the probability of ant $k$ jumping from node $i$ to node $j$ at the $t$-th time of traversal search, $\psi_{i j}(t)$ represents the pheromone concentration of the path between node $i$ and $j$ at the $t$-th time of traversal search, $f_{i j}(t)$ and $g_{i j}(t)$ are the energy metric parameters of node $i$ and $j$ at the $t$-th time of traversal search, $h_{i j}(t)$ represents the space parameter of node $i$ and $j$ at the $t$-th time of traversal search, $j \in$ allowed $_{k}$ 
represents that node $j$ belongs to the set that does not include the nodes that ant $k$ has passed within the wireless communication range of node $i, s \in C(i)$ represents that node $s$ belongs to the node set of node $i$ within the wireless communication scope, $e_{j}(t)$ represents the energy that node $j$ has at the $t$-th time of traversal search, $E_{\max }$ represents the maximum energy that can be provided by node, $h_{j d}^{-1}$ represents the rely hop count between node ${ }^{j}$ and goal node $d$, and $\alpha, \beta, \gamma$ and $\delta$ are importance factors of corresponding parameters.

When all ants arrive at the goal node according to the selection probability, pheromones on all paths are updated. The update formula [13] is as follows:

$$
\left\{\begin{array}{l}
\psi_{i j}(t+1)=(1-\rho) \psi_{i j}(t)+\Delta \psi_{i j}(t) \\
\Delta \psi_{i j}(t)=\sum_{k=1}^{n} \frac{c E_{\text {avg }}^{k}\left(h_{\max }-h_{k}\right)}{w h_{k}}
\end{array}\right.
$$

where $\rho$ is the pheromone volatilization coefficient, $\Delta \psi_{i j}(t)$ is the increment of pheromone between node $i$ and ${ }^{j}, c$ is a constant, $w$ is a normal number, $E_{\text {avg }}^{k}$ is the node average energy of ant $k$ in the current path, $h_{\text {max }}$ is the maximum node hop count that ant in the network can realize, and $h_{k}$ is the node hop count of ant $k$ in the current path. After the pheromone is updated, the ant colony is asked to search the path again. The above steps are repeated until the termination condition is reached, and the path with the most pheromones is regarded as the optimal path.

\subsection{Improved ant colony algorithm}

In the process of calculating the selection probability of the next-hop node of an ant by the ant colony algorithm described above, although it takes into account the sharing of node energy efficiency so that the path can achieve the optimal energy efficiency, in the actual VANET, the premise of successful communication between nodes is that the nodes are connected, and the links between nodes may be broken as the vehicles acting as nodes are constantly changing in VANET. Only considering the energy efficiency, the nodes in the optimal path are likely to be disconnected. Therefore, this study improved the ant colony algorithm, predicted the connectivity by connectivity probability, put it into the calculation of node selection probability, and optimized the node searching of the ant colony. The process of the improved ant colony algorithm is shown in Figure 2.

(1) Parameters are initialized.

(2) Starting from the initial node, each ant chooses the next node according to the improved probability formula.

The formula of the improved selection probability is:

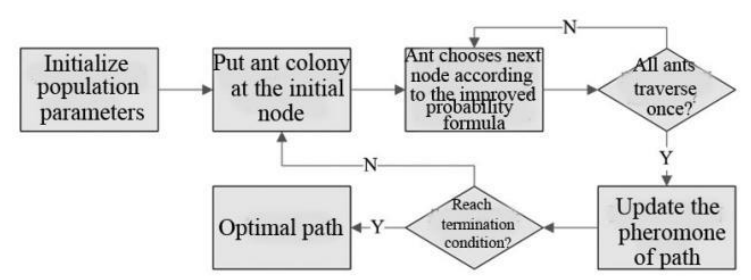

Figure 2: The process of the improved ant colony algorithm.

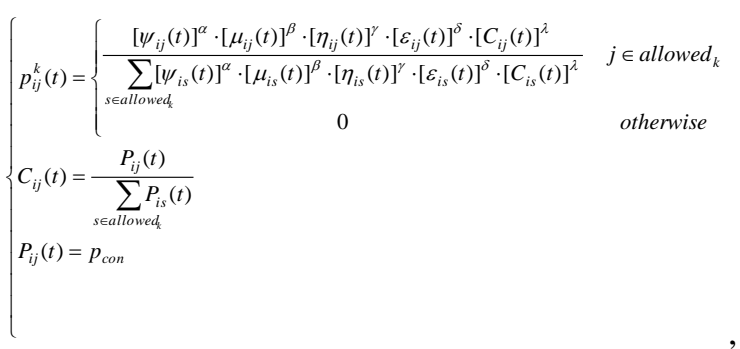

where $C_{i j}(t)$ represents for the metric parameter of connectivity between node $i$ and $j$ and $\lambda$ is an importance factor of the corresponding parameter.

(3) Whether all ants in the ant colony have traversed from the initial node to the target node once is determined. If not, step (2) repeats; if they do, then the pheromone in the path that the ant passes through is updated, and the update formula is equation (3).

(4) Whether the algorithm has reached the termination condition is determined. If it does, it outputs the optimal path; if not, it will return to step (2). The termination conditions include reaching the maximum number of iterations (taking all ants in the ant colony traversing the path once and updating the pheromone as one time of iteration) and the ant selection path converging to stability.

\section{Simulation experiment}

\subsection{Experimental environment}

In this study, the running track and running state of vehicles were simulated by VANET mobile simulator, and the network which was composed of vehicles and the routing algorithm proposed were simulated by MATLAB software [14]. VANET mobile simulator was a free software developed based on the Java platform, which can realize the macro and micro-movement simulation of vehicles, and it can make the movement of simulated vehicles closer to the actual situation. MATLAB software is a network simulation software, which builds the network based on the vehicle trajectory simulated by the VANET simulator and simulates the routing algorithm. The experiment was carried out in a server in a laboratory. The server configuration was Windows 7 system, i7 processor, and $16 \mathrm{G}$ memory. 


\subsection{Experimental parameters}

The relevant parameters of the simulation experiment are shown in Table 1, including vehicle simulation parameters and network simulation parameters. Vehicle simulation parameters included road topology size, number of vehicles, speed range, vehicle acceleration, and lane number. Network simulation parameters included simulation time, number of ant colonies, node communication radius, pheromone volatilization coefficient, minimum pheromone, $E_{\max }$, node unit information transmitting and receiving energy and node communication protocol. Also, the Nakagami channel transmission model was adopted for the channel attenuation in the process of node communication [15]. To verify the performance of the improved algorithm, it is compared with the ant colony algorithm and geographic location-based routing algorithm.

\subsection{Performance indicators}

The indicators that can reflect the reliability of network data transmission include packet loss rate and time delay. Packet loss refers to the phenomenon that part of the data packets are lost due to the instability or disconnection of the node-link when the information is transmitted to other nodes in the form of data packets. The packet loss rate is the degree of packet loss. Time delay is the time taken for data transmission from one node to the goal node. For the node network, the lower the packet loss rate and time delay are, the faster and more stable data transmission is and the more reliable the whole network is. In this study, the network delay and packet loss rate were estimated by the time stamp method.

In addition to the above two indicators, the reliability of network transmission can also be measured by the statistical method. In this study, the VANET under three routing algorithms were simulated and analyzed by the Monte Carlo method many times, and the number of times that did not meet the requirements was counted to estimate the reliability of the network. The estimation formula is:

$$
R_{i}=1-\frac{N_{1}}{i},(5)
$$

where $R_{i}$ stands for the estimated value of reliability after the $i$-th time of the simulation and $N_{1}$ stands for the number of times that the network indicators did not meet the requirements after the $i$-th time of simulation. When the network transmits data, if the average packet loss rate was greater than $4 \%$ or the average delay was greater than $100 \mathrm{~ms}$, it was considered that the network indicators of this simulation did not meet the requirements. In this study, when the Monte Carlo method was used, the precision interval was $\pm 3 \%$, and $97 \%$ of them met the precision interval.

\subsection{Experimental results}

As shown in Figure 3, under the same number of vehicle nodes, the packet loss rate of the algorithm proposed in this study was the lowest, and the routing algorithm based

\begin{tabular}{|c|c|c|}
\hline \multirow{5}{*}{$\begin{array}{l}\text { Vehicle } \\
\text { simulation } \\
\text { parameters }\end{array}$} & $\begin{array}{l}\text { Road topology } \\
\text { size }\end{array}$ & $1000 m \times 1000 m$ \\
\hline & $\begin{array}{l}\text { Number of } \\
\text { vehicles }\end{array}$ & $10 \sim 100$ \\
\hline & Speed range & $10 \sim 30 \mathrm{~m} / \mathrm{s}$ \\
\hline & $\begin{array}{l}\text { Vehicle } \\
\text { acceleration }\end{array}$ & $2 m / s^{2}$ \\
\hline & $\begin{array}{l}\text { Number of } \\
\text { lanes }\end{array}$ & 3 \\
\hline \multirow{9}{*}{$\begin{array}{l}\text { Network } \\
\text { simulation } \\
\text { parameters }\end{array}$} & Simulation time & $900 \mathrm{~s}$ \\
\hline & $\begin{array}{l}\text { Number of ant } \\
\text { colonies }\end{array}$ & 30 \\
\hline & $\begin{array}{l}\text { Node } \\
\text { communication } \\
\text { radius }\end{array}$ & $150 \mathrm{~m}$ \\
\hline & $\begin{array}{l}\text { Pheromone } \\
\text { volatilization } \\
\text { coefficient }\end{array}$ & 0.7 \\
\hline & $\begin{array}{l}\text { Minimum } \\
\text { pheromone }\end{array}$ & 0.01 \\
\hline & $E_{\max }$ & $1 \mathrm{~J}$ \\
\hline & $\begin{array}{l}\text { Node unit } \\
\text { information } \\
\text { transmitting } \\
\text { energy }\end{array}$ & $4.3 \mu J / b i t$ \\
\hline & $\begin{array}{l}\text { Node unit } \\
\text { information } \\
\text { receiving } \\
\text { energy }\end{array}$ & $2.4 \mu \mathrm{J} / \mathrm{bit}$ \\
\hline & $\begin{array}{l}\text { Node } \\
\text { communication } \\
\text { protocol }\end{array}$ & IEEE 802.11 \\
\hline
\end{tabular}

Table 1: Related parameters of the simulation experiment.

on geographical location had the highest packet loss rate It was seen from the curve variation that the network packet loss rate under the three routing algorithms showed a trend of decreasing first and then increasing with the increase of vehicle nodes in the network, and the number

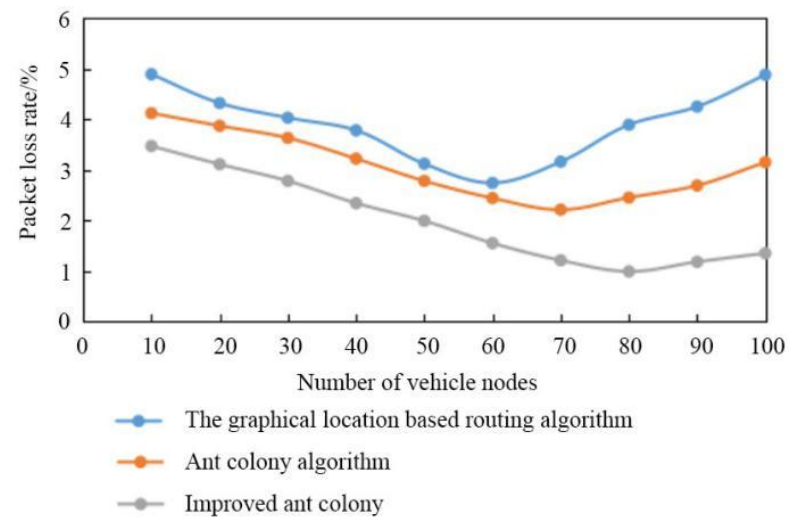

Figure 3: Relationship between network packet loss rate and nodes under three routing algorithms. 
of vehicle nodes of the improved algorithm was the largest and that of the routing algorithm based on geographical location was the smallest when the packet loss rate was the lowest.

As shown in Figure 4, under the same number of vehicle nodes, the network average delay of the algorithm proposed in this study was the smallest, and that using of the routing algorithm based on geographical location was the largest. The average delay curve changes of the three routing algorithms clearly showed that the average delay of the network first decreased and then increased with the increase of the number of vehicle nodes, and the number of vehicle nodes of the ant colony algorithm and improved ant colony algorithm was the largest when the average time delay was the smallest and increased gently afterward.

The simulation model was simulated by the Monte Carlo method for many times, and the simulation times that did not meet the set indicators were counted to evaluate the reliability of the network. The results are shown in Figure 5. It was seen from Figure 5 that the network reliability of the improved ant colony algorithm was the highest, and the network reliability of the routing algorithm based on geographical location was the lowest under the same number of vehicle nodes. It was seen from the curve changes that the network reliability of the routing algorithm based on geographical location and ant colony algorithm increased first and then decreased, while the network reliability of the improved ant colony algorithm increased first and then basically stabilized at 0.99 .

The above three results showed that the improved ant

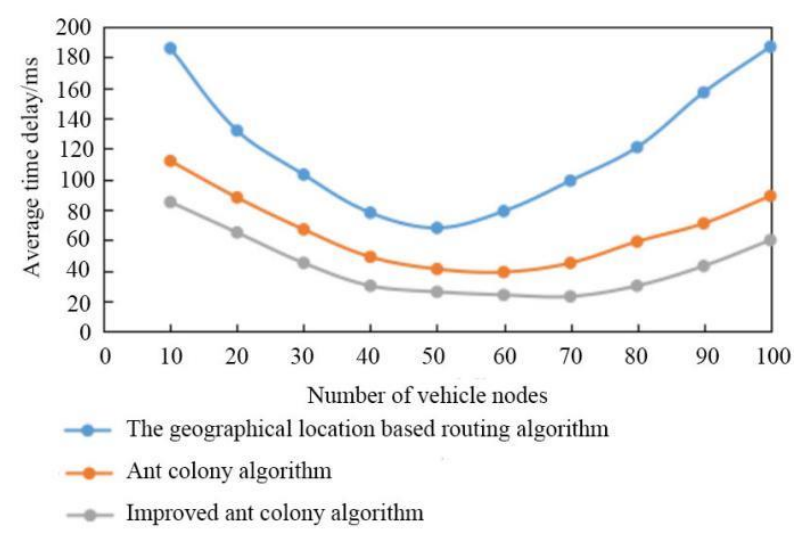

Figure 4: Relationship between average network delay and nodes under three routing algorithms.

colony algorithm could effectively reduce the packet loss rate and average delay in the data transmission between nodes in VANET and improve the reliability of network data transmission. Although the packet loss rate and average delay of the network increased when the number of vehicles in VANET increased to a certain extent, under the effect of the improved ant colony algorithm, the increase amplitude was smaller than the other two

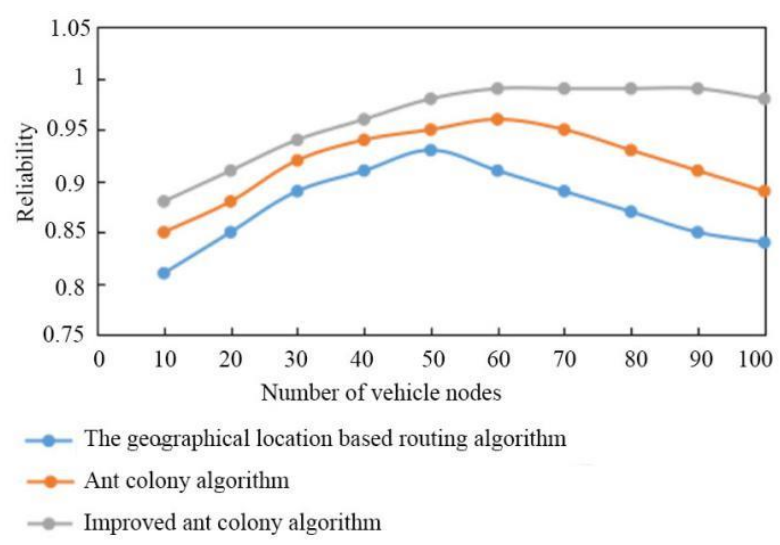

Figure 5: Relationship between network reliability and nodes under three routing algorithms.

algorithms, and the reliability of network transmission was maintained at a high level. The reason why the packet loss rate and time delay decreased and the reliability increased was that the number of nodes that could transmit smoothly around the nodes increased when the number of vehicle nodes in the network increased, which led to more choices of the data transmission path. The reason why the packet loss rate and time delay increased and the reliability decreased was that when the vehicle nodes increased to a certain extent, the interference of the surrounding node signals increased although the optional excellent path increased. However, the improved ant colony algorithm not only considered the energy efficiency but also considered the connectivity between vehicles, which made the path selection more inclined to the path with stronger connectivity to slow down the increase of packet loss rate and delay and maintain the stability of reliability.

\section{Conclusion}

This paper briefly introduced the vehicle communication network, VANET, and the ant colony algorithm for path search in the network, improved the ant colony algorithm, carried out a simulation experiment on the improved ant colony algorithm on the simulation platform, and compared it with the traditional ant colony algorithm and the geographical location-based routing algorithm. The results showed that: (1) with the increase of vehicle nodes in VANET, the packet loss rate presented a tendency of increasing first and then decreasing, the packet loss rate of the improved ant colony algorithm was always the lowest, and the packet loss rate of the geographical location-based routing algorithm was always the highest; (2) with the increase of vehicle nodes, the average delay in the network firstly decreased and then increased, the improved ant colony algorithm was always the smallest, and the geographical location based routing algorithm was always the largest; (3) the reliability of network data transmission increased and then decreased with the increase of nodes, but the reliability of the network under the improved ant colony algorithm rose first and then tended to be stable, the network reliability under the improved ant colony algorithm was always the highest and that of the routing 
algorithm based on geographical location was always the lowest.

\section{References}

[1] Bitam S, Mellouk A, Zeadally S (2015). VANETcloud: a generic cloud computing model for vehicular Ad Hoc networks. IEEE Wireless Communications, 22(1), pp. 96-102.

[2] Amoozadeh M, Deng H, Chuah CN, Zhang HM, Ghosal D (2015). Platoon management with cooperative adaptive cruise control enabled by VANET. Vehicular Communications, 2(2), pp. 110123. https://doi.org/10.1016/j.vehcom.2015.03.004

[3] Melaouene N, Romadi R (2019). An enhanced routing algorithm using ant colony optimization and VANET infrastructure. MATEC Web of Conferences, 259(6), pp. 02009. https://doi.org/

[4] Kumar N, Dave M (2016). BIIR: A Beacon Information Independent VANET Routing Algorithm with Low Broadcast Overhead. Wireless Personal Communications, 87(3), pp. 869-895. https://doi.org/10.1007/s11277-015-2620-y

[5] Saxena R, Jain M, Sharma DP, Jaidka S, Thampi SM, El-Alfy EM (2019). A review on VANET routing protocols and proposing a parallelized genetic algorithm based heuristic modification to mobicast routing for real time message passing. Journal of Intelligent \& Fuzzy Systems, 36(3), pp. 2387-2398. https://doi.org/10.3233/jifs-169950

[6] Li N, Martinez-Ortega JF, Diaz VH, Fernandez JAS (2017). Probability Prediction based Reliable Opportunistic (PRO) Routing Algorithm for VANETs. IEEE/ACM Transactions on Networking, PP(99). https://doi.org/10.1109/TNET.2018.2852220

[7] Li AN, Zhao YM, Xie P, Wang Q (2016). Research on routing algorithm based on the VANET. MATEC Web of Conferences, 44, pp. 01085-. https://doi.org/10.1051/matecconf/20164401085

[8] Kumuthini C, Krishnakumari P (2015). An access point based routing algorithm (APBR) using greedy method towards improving quality of service for VANET. International Journal of Applied Engineering Research, 10(3), pp. 5489-5502.

[9] Saravanan M, Ganeshkumar P, Kumar SM (2018). Survey on opportunistic routing algorithm for Vehicular Adhoc Network (VANET). International Journal of Pure and Applied Mathematics, 118(20), pp. 1735-1740.

[10] Aadil F, Bajwa KB, Khan S, Chaudary NM, Akram A (2016). CACONET: Ant Colony Optimization (ACO) Based Clustering Algorithm for VANET. Plos One, 11(5), pp. e0154080.

https://doi.org/10.1371/journal.pone.0154080

[11] Goudarzi F, Asgari H, Al-Raweshidy HS (2018). Traffic-aware VANET routing for city environmentsA protocol based on ant colony optimization. IEEE Systems Journal, pp. 1-11.
[12] Deepa Thilak K, Amuthan A (2017). Cellular Automata-based Improved Ant Colony-based Optimization Algorithm for mitigating DDoS attacks in VANETs. Future Generation Computer Systems, 82(MAY), pp. 304-314. https://doi.org/10.1016/j.future.2017.11.043

[13] Goudarzi F, Asgari H, Al-Raweshidy HS (2019). Traffic-Aware VANET Routing for City Environments-A Protocol Based on Ant Colony Optimization. IEEE Systems Journal, 2019, 13(1), pp. 571-581.

[14] Jindal V, Bedi P (2017). Preemptive MACO (MACOP) Algorithm for Reducing Travel Time in VANETs. Applied Artificial Intelligence, pp. 74-196. https://doi.org/10.1080/08839514.2017.1300017

[15] Lakshmanaprabu SK, Shankar K, Rani SS, Abdulhay E, Arunkumar N, Ramirez G, Uthayakumar J (2019). An effect of big data technology with ant colony optimization based routing in vehicular ad hoc networks: Towards smart cities. Journal of Cleaner Production, 2019, 217(APR.20), pp. 584-593. https://doi.org/10.1016/j.jclepro.2019.01.115 